\title{
Centrilobular ductular reaction correlates with fibrosis stage and fibrosis progression in non-alcoholic steatohepatitis
}

\author{
Lei Zhao ${ }^{1}$, Maria Westerhoff ${ }^{2}$, Rish K Pai ${ }^{3}$, Won-Tak Choi ${ }^{4}$, Zu-Hua Gao ${ }^{5}$ and John Hart ${ }^{6}$ \\ ${ }^{1}$ Department of Pathology, Brigham and Women's Hospital, Boston, MA, USA; ${ }^{2}$ Department of Pathology, \\ University of Michigan, Ann Arbor, MI, USA; ${ }^{3}$ Departments of Laboratory Medicine and Pathology, Mayo \\ Clinic, Scottsdale, AZ, USA; ${ }^{4}$ Department of Pathology, University of California at San Francisco, San \\ Francisco, CA, USA; ${ }^{5}$ Department of Pathology, McGill University, Montreal, QC, Canada and ${ }^{6}$ Department of \\ Pathology, University of Chicago, Chicago, IL, USA
}

\begin{abstract}
There is increasing interest in the role of ductular reaction as part of the pathogenesis and characteristic histology of non-alcoholic steatohepatitis. However, earlier studies did not separately assess the contribution of periportal and centrilobular zone ductular reaction over the spectrum of non-alcoholic steatohepatitis, and their clinical significance remains unclear. We herein analyzed the character of ductular reaction in each hepatic zone in non-alcoholic steatohepatitis biopsies and for the first time evaluated the prognostic value of ductular reaction in baseline biopsies as a predictor of progression of fibrosis in subsequent biopsies. A total of 90 non-alcoholic steatohepatitis liver biopsies were included in the cohort. The relationships among ductular reaction, grade, stage, and other common histopathologic findings in non-alcoholic steatohepatitis were analyzed in a crosssectional manner. Among these patients, a total of 47 patients underwent sequential liver biopsies in the absence of effective treatment. The frequency of ductular reaction and the other histopathologic parameters in the initial biopsies were analyzed as predictors of progression of fibrosis in the second biopsies in a longitudinal analysis. Centrilobular ductular reaction was identified in $90 \%$ of patients and $38 \%$ of centrilobular zones. The prevalence of centrilobular ductular reaction increased as non-alcoholic steatohepatitis grade increased $(P=0.0002)$ and also as stage of fibrosis increased $(P<\mathbf{0 . 0 0 0 1})$ in the cross-sectional study. In the longitudinal study, the frequency of centrilobular ductular reaction in the initial biopsies was significantly higher in the group of progressors and correlated with the rate of fibrosis progression $(P=0.02)$. Centrilobular ductular reaction is common in non-alcoholic steatohepatitis and its presence correlates significantly with increasing necroinflammatory activity and fibrosis stage. Development of centrilobular ductular reaction appears to predict progression of fibrosis in subsequent biopsies. Modern Pathology (2018) 31, 150-159; doi:10.1038/modpathol.2017.115; published online 1 September 2017
\end{abstract}

Liver biopsy histology plays an important role in the clinical management of non-alcoholic fatty liver disease and remains the gold standard for diagnosing and monitoring of non-alcoholic steatohepatitis. ${ }^{1}$ Steatosis, hepatocellular ballooning degeneration, and lobular inflammation are the hallmark findings of adult non-alcoholic steatohepatitis histologically. ${ }^{2-4}$ When liver injury persists, characteristic centrilobular sinusoidal fibrosis may develop, followed by portal and periportal fibrosis in some

Correspondence: Professor J Hart, MD, Department of Pathology, University of Chicago, 5841 S. Maryland Avenue, E-607D, MC 6101, Chicago, IL 60637, USA.

E-mail: john.hart@uchospitals.edu

Received 18 October 2016; revised 17 July 2017; accepted 18 July 2017; published online 1 September 2017 cases. Eventually centro-portal and centro-central bridging fibrosis develop, which ultimately leads to micronodular cirrhosis. Between 10 and 29\% of individuals with non-alcoholic steatohepatitis develop cirrhosis within 10 years. ${ }^{5,6}$ However, none of the aforementioned characteristic histologic changes seems to effectively predict the progression of fibrosis in subsequent biopsies in long-term follow-up studies. ${ }^{5,7-17}$ Therefore, identification of additional histologic features that may better represent the biological events mediating hepatic injury and fibrosis would be of great value in improving the predictive value of histologic grading of nonalcoholic steatohepatitis.

Ductular reaction is intimately associated with fibrosis and its role in non-alcoholic steatohepatitis 
has been a topic of increasing interest. ${ }^{18-20}$ Ductular reaction refers to the proliferation of ductular type epithelium $^{21}$ and is believed to represent activation and proliferation of hepatic progenitor cells in response to biliary or significant hepatocellular injury. ${ }^{22}$ Reactive ductular epithelium has shown to promote periportal fibrosis, neoangiogenesis, and inflammation through immune modulators and by direct activation of multiple signaling pathways in animal models of biliary diseases. ${ }^{23,24}$ Periportal ductular reaction is also reported in chronic hepatitic $\mathrm{C}$ and other chronic liver diseases, and is speculated to be closely associated with the development of portal and periportal fibrosis in these conditions. ${ }^{18,19,25-27}$

Until recently, ductular reaction had only been described in the periportal compartment, where the canals of Herring, the residence of hepatic progenitor cells, are located. However, a few recent studies have reported the presence of ductular type epithelium in the centrilobular zone in adult non-alcoholic steatohepatitis. ${ }^{20,28}$ As both hepatocyte injury and fibrosis are accentuated in the centrilobular zone in non-alcoholic steatohepatitis, it is reasonable to hypothesize that the centrilobular ductular reaction may be an important process that mediates hepatic injury and fibrosis. However, the studies to date have not provided quantitative analysis of centrilobular ductular reaction in a systematic manner. Moreover, there has been no investigation on prognostic value of centrilobular ductular reaction.

In this study we set out to investigate the clinical implication of ductular reaction in each hepatic zone in adult non-alcoholic steatohepatitis by analyzing the association between ductular reaction and fibrosis in cross-sectional and longitudinal settings. To ensure accurate quantitation, immunostains for cytokeratin 7 (CK7), a cytokeratin that is present in ductular type epithelium, and glutamine synthetase, an enzyme that is expressed only by centrilobular (zone 3) hepatocytes, ${ }^{29-31}$ were used for identification of ductular type epithelium and to separate the different hepatic zones, respectively (Figure 1).

\section{Materials and methods}

\section{Patients and Clinical Data}

Liver biopsies from adult patients (age $\geq 17$ years) with a primary pathologic diagnosis of non-alcoholic steatohepatitis were selected from four centers (University of Chicago, University of Washington, Cleveland Clinic and McGill University). Cases with a documented history of liver transplantation, superimposed HCV infection or other chronic liver diseases, alcohol abuse, and hepatic venous outflow obstruction were excluded from the study. Cases with cirrhosis were also excluded. Ninety patients met the above criteria. Among them, 47 patients who did not receive any treatment (including gastric

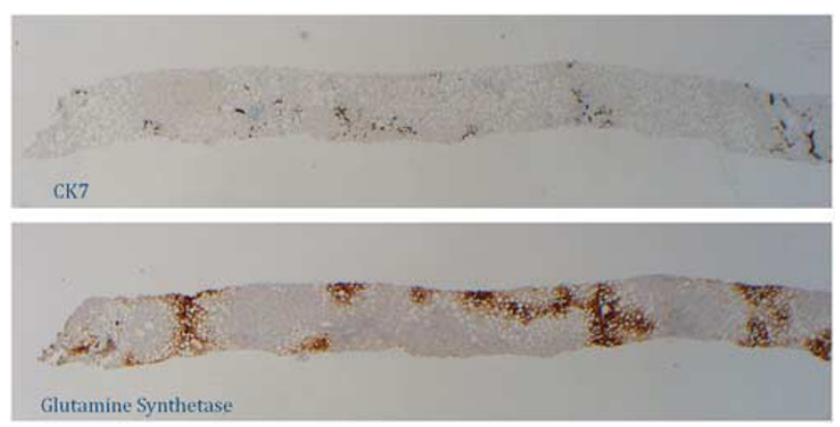

Figure 1 Quantitation of ductular reaction in centrilobular zone and periportal zone. Glutamine synthetase immunostain was used to highlight centrilobular zone (lower panel) and CK7 immunostain was used to facilitate identification of ductular epithelium (upper panel) (photos taken at $2 \times$ objective).

bypass) had a subsequent biopsy performed at least 12 months later. Clinical and laboratory data were collected retrospectively from electronic medical records. The study was approved by the Institutional Review Board of each of the above-mentioned institutions.

A group of 19 patients with HCV hepatitis were also included as the control group. None of these patients had superimposed steatosis or nonalcoholic steatohepatitis.

\section{Morphologic Analysis}

Biopsy slides were reviewed masked as to patient clinical data. The grade of activity was assigned using the modified Brunt ${ }^{3}$ system and stage of fibrosis was scored using Kliener ${ }^{4}$ staging system. The common histologic features of adult nonalcoholic steatohepatitis were separately scored as follows: hepatocellular ballooning degeneration: 0: none to rare ballooned cells; 1 : few ballooned cells; 2: many ballooned cells; Mallory-Denk body: 0: none; 1: few; 2: many; Extent of macrovesicular steatosis: 1: 5-33\%; 2: 34-66\%; 3: >66\%; lobular inflammation: 0: No foci; 1 : $1-2$ foci per $20 \times$ field; 2 : 2-4 foci per $20 \times$ field; $3:>4$ foci per $20 x$ field; portal inflammation: 0 : none; 1: mild; 2 : moderate; 3: severe; neutrophils: 0: none; 1 : few/occasional; 2 : many/frequent; hepatocellular iron $(0-4+)$; location of steatosis: centrilobular, non-centrilobular (periportal or panlobular); fibrosis stage: 0: no fibrosis; 1A: perisinusoidal delicate fibrosis; 1B: perisinusoidal dense fibrosis; 1C: portal and or periportal fibrosis only; 2: perisinusoidal and portal/periportal fibrosis; 3: bridging fibrosis; 4: cirrhosis. In statistical analysis, stage $1 \mathrm{~A}$ is counted as 0.5 , due to significant less fibrosis in stage $1 \mathrm{~A}$ than stage $1 \mathrm{~B} / \mathrm{C}$.

\section{Immunohistochemistry}

Immunostains for cytokeratin 7 (CK7) (Dako, Carpentaria, CA, USA) and glutamine synthetase 
(BD, San Jose, CA, USA ) were performed on two consecutive sections of each biopsy case utilizing an automated stainer (Bond automated IHC stainer). The degree of ductular reaction within each glutamine synthetase-positive centrilobular zone of every biopsy was recorded as either: 0: no CK7+ cells, 1: isolated single CK7+ cells, 2: CK7+ cells in strings, or 3: CK7+ ductular structures (Figure 1). In addition every portal tract in the CK7 stained slides was graded as either: 0: no ductular reaction, 1: mild ductular reaction, or 2: florid ductular reaction.

\section{Statistics}

The difference between groups with ordinal data was compared by Mann-Whitney tests. The difference between groups with normally distributed variables was compared by Student's $t$-test. The degree of association between ordinal variables was determined by Spearman rank order test. Multivariate analysis was performed to analyze the association between centrilobular ductular reaction versus periportal ductular reaction and grade and stage. Nonlinear univariate analysis was performed to evaluate the correlation between histological parameters, frequency of ductular reaction with the rate of fibrosis progression. All analyses were performed using EViews 8.0 (IHS Global Inc., Engelwood, Colorado, California, USA).

\section{Results}

\section{Cross-Section Study of Centrilobular Ductular Reaction in Non-Alcoholic steatohepatitis}

Patient populations. A total of 90 patients with a diagnosis of non-alcoholic steatohepatitis were included in the study. None of the patients had a documented history of liver transplant, alcohol abuse, hepatic venous outflow obstruction or superimposed HCV infection. All biopsies were performed before any medical intervention. Patient age ranged from 33 years to 78 years, with a median of 54 years. There were 45 men and 45 women.

Histopathologic analysis. Of the 90 liver biopsies, 22 exhibited no evidence of fibrosis (Brunt fibrosis stage 0), 27 exhibited centrilobular sinusoidal fibrosis only (Brunt fibrosis stage 1), 24 demonstrated both centrilobular and periportal fibrosis (Brunt fibrosis stage 2) and the remaining 17 cases showed centro-portal and centro-central bridging fibrosis (Brunt fibrosis stage 3). Cases with cirrhosis were excluded from the study due to the complete disarrangement of metabolic zonation. The normal pattern of centrilobular perivenular staining with glutamine synthetase was maintained in all study cases. In some cases the degree of centrilobular reactivity with glutamine synthetase was somewhat greater that in normal liver in terms of the width of the positive zone, and in a few case it was somewhat diminished. However, in all cases the centrilobular zones were clearly delineated. A majority of the cases exhibited mild or moderate disease activity (44\% with Brunt activity grade 1 and $47 \%$ with Brunt activity grade 2).

Characteristics of ductular reaction in non-alcoholic steatohepatitis. A total of 2225 centrilobular zones were analyzed from the 90 biopsies. Only small cells with unequivocal biliary morphology and strong CK7 immunoreactivity were counted. Three forms of centrilobular ductular reaction were identified: (1) scattered single CK7-positive cells, (2) strings or a single plate of CK7-positive cells, and (3) well formed CK7-positive ductules with lumens (Figures 1-3).

CK7-positive structures (any of the above three forms) were identified in $90 \%$ of biopsies and $38 \%$ of the centrilobular zones. The frequency of centrilobular ductular reaction increased as stage of fibrosis increased, with a statistically significant positive correlation $(P$-value $<0.0001)$ (Figure 4; Table 1). Of note, even in some of the stage 0 biopsies, there was already significant centrilobular CK7-positive ductular epithelium that preceded the development of any fibrosis. The frequency of centrilobular ductular reaction also increased as the grade of activity increased (Figure 4). There was positive correlation between centrilobular ductular reaction and the severity of lobular inflammation, hepatocellular ballooning degeneration, presence of Mallory-Denk bodies, and the overall Brunt grade $(P$ value: $0.058,0.007,0.005$, and 0.0002 , respectively) (Table 1).

A total of 1598 portal tracts were analyzed, and $82 \%$ of the portal tracts contained either a mild or moderate ductular reaction (Figure 4). There were also correlations between the presence of a periportal ductular reaction and stage, grade, degree of hepatocellular ballooning degeneration, presence of Mallory-Denk bodies, and portal inflammation (Figure 4; Table 1). However, when analyzed in a multivariate regression model, centrilobular ductular reaction showed a much stronger correlation with stage of fibrosis $(P<0.00001$ vs $P=0.081)$ (Table 2$)$, and the correlation between periportal ductular reaction and grade became insignificant $(P=0.541)$ (Table 2).

To investigate whether centrilobular ductular elements were also present in other chronic liver diseases, a control group of 19 patients with chronic HCV hepatitis and stage 0-2 fibrosis were scored using the same methodology. The results were compared with 67 non-alcoholic steatohepatitis patients who also had stage $0-2$ fibrosis. The frequency of centrilobular ductular elements was significantly higher in non-alcoholic steatohepatitis than in HCV patients (Figure 5). 

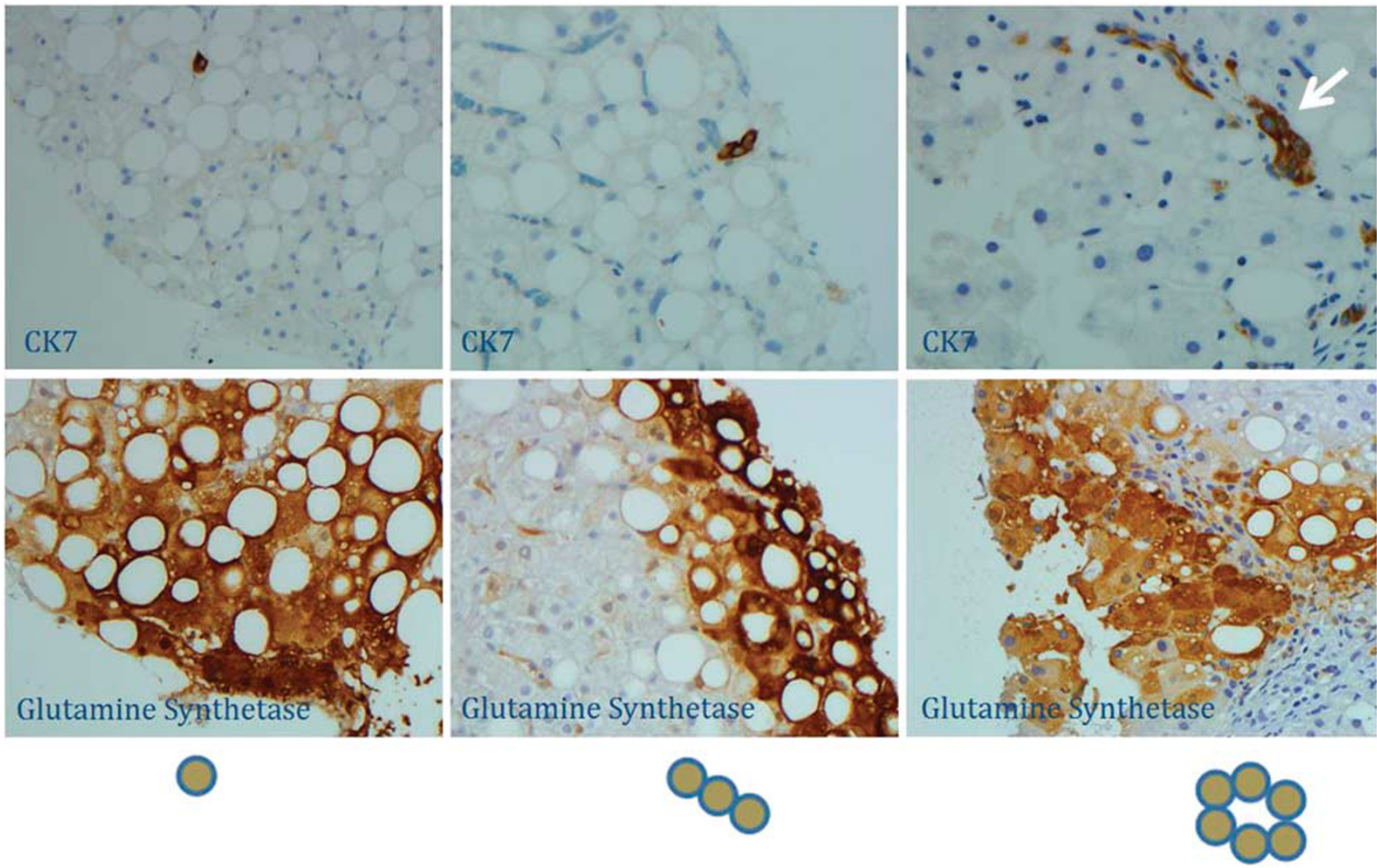

Figure 2 Examples of centrilobular CK7-positive elements. Glutamine synthetase highlights the centrilobular zones (lower panels). CK7positive single cells, string of cells and ductules with lumens are shown in left upper, middle upper, and right upper panel, respectively (photos taken at $20 \times$ objective).

\section{Longitudinal Study of Centrilobular Ductular Reaction and Fibrosis Progression}

Patient characteristics and histopathologic features at baseline. A subset of 47 of the 90 patients included in the study had undergone a subsequent liver biopsy at least 12 months after the initial one. No medical or surgical treatment was given between the two biopsies. Patient age ranged from 17 to 65 years, with a median of 51. There were 26 men and 21 women. Among the 47 patients, 21 increased in fibrosis stage while 26 patients remained the same or regressed (Table 3).

The histologic features present in the initial biopsies are summarized in Table 4. The majority of these biopsies demonstrated no or mild fibrosis (with $32 \%$ patients being stage $0,47 \%$ being stage $1,13 \%$ being stage 2 , and $9 \%$ being stage 3 ). The degree of steatosis ranged from mild to severe, with each of the three categories well represented in the cohort. Most cases showed mild to moderate lobular inflammation with no or few neutrophils. Portal inflammation was usually mild or absent. Hepatocellular ballooning degeneration was common but Mallory-Dank bodies were rare.

Comparison of histopathologic features and ductular reaction at baseline between fibrosis progressors and non-progressors. Patients who developed increased fibrosis stage in the follow-up biopsy (progressors) were compared with patients with stable or regressed fibrosis stage (non-progressors). As listed in Table 5, degree or location of steatosis, lobular inflammation, portal inflammation, ballooning, Mallory-Denk body formation, neutrophils, or iron deposition did not differ significantly between the two groups.

The frequency of centrilobular ductular reaction (including single cell form, strings of cell, and well formed ductules and the sum of all three forms) was significantly higher in the progressor group (Table 5; Supplementary Table 1). In contrast, the frequency of portal ductular reaction did not show significant difference between the groups of progressors and non-progressors. Furthermore, a univariate nonlinear regression analysis showed that among all of the above listed histologic variables, only the frequency of centrilobular CK7-positive elements was positively correlated with the rate of fibrosis progression (difference of fibrosis divided the time interval in years) with a $P$-value $<0.05$ (Table 6).

\section{Correlation With Clinical Parameters}

Available clinical parameters including age, gender, presence or absence of obesity or diabetes, and serum transaminase levels were collected through 

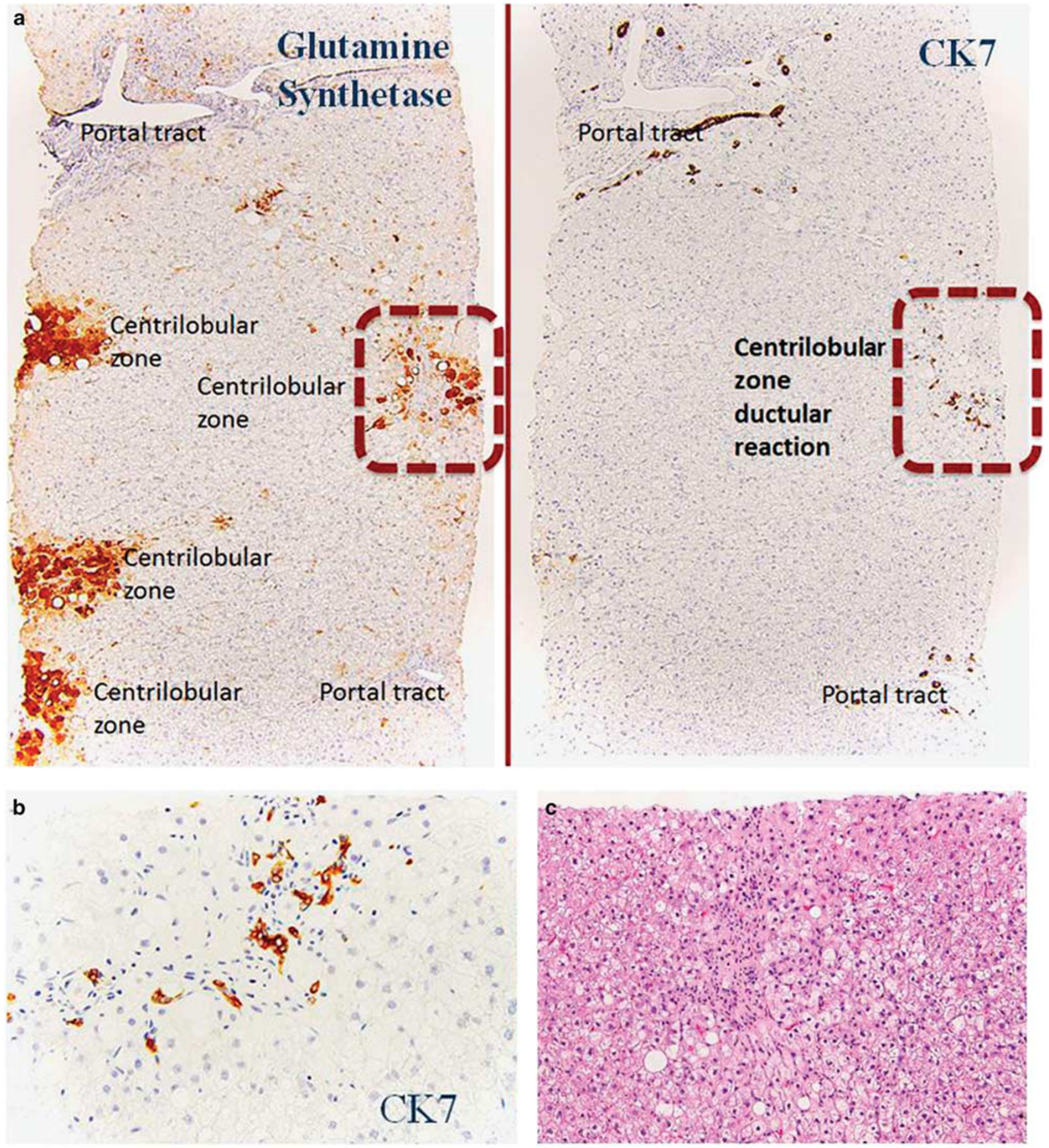

Figure 3 Example of centrilobular ductular reaction. In this example, glutamine synthetase immunostain highlights centrilobular zone. CK7 stains ductular reaction in both centrilobular zone and portal tracts. The area with centrilobular zone ductular reaction is circled and enlarged in $\mathbf{b}$. The corresponding H\&E staining of the same area is shown in $\mathbf{c}$ (photos taken at $4 \times$ objective (a) and $10 \times$ objective (b and $\mathbf{c})$ ).

review of the electronic medical records. The 47 patients who had undergone a subsequent liver biopsy seemed to be younger in age $(P$-value $=0.049)$ and had lower aspartate transaminase level $(P$-value $=0.013)$ than the patients who did not receive repeat biopsies (Table 7).
Upon analysis of clinical features between the groups of progressors and non-progressors, the only statistically significant finding was that the serum aspartate transaminase level was higher in the progressor group (with a mean value of 67 IU) than that of the non-progressor group (which has a mean 

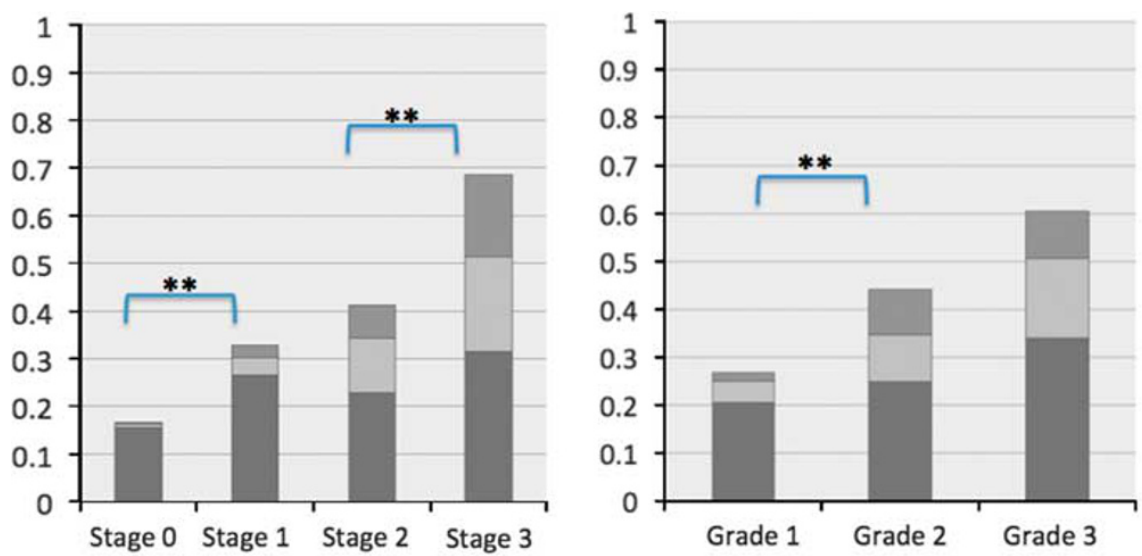

\section{Centrilobular CK7+ ductules}

\section{Centrilobular string of CK7+ cells}

\section{Centrilobular single CK7+ cells}
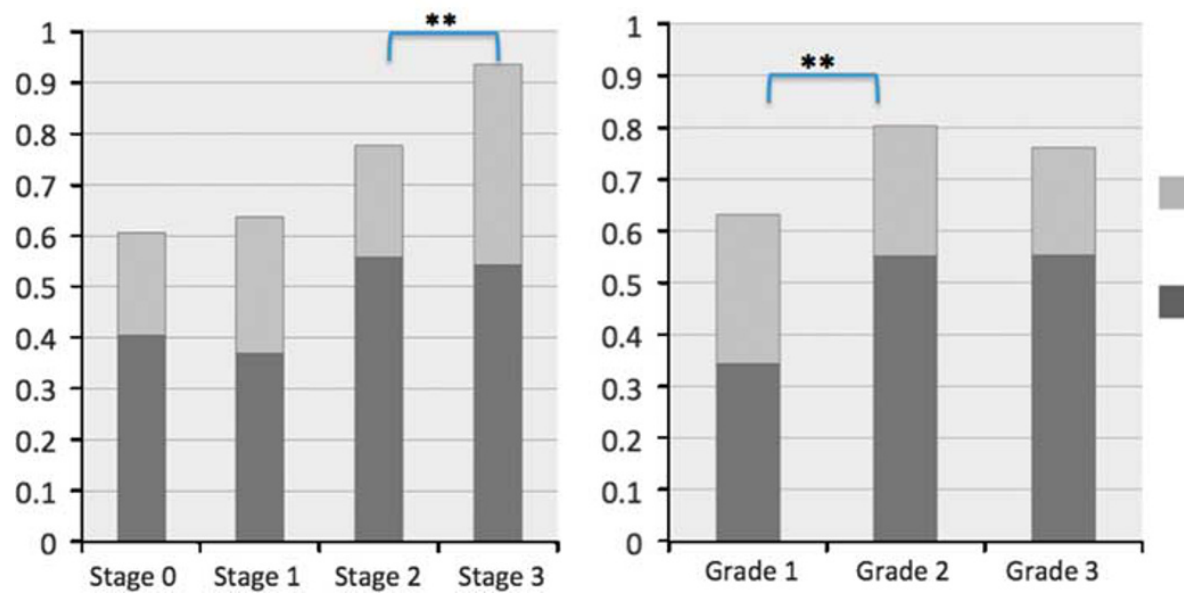

Portal tract florid ductular reaction

Portal tract mild ductular reaction

Figure 4 Ductular reaction in cross-sectional study. The correlation between centrilobular ductular reaction and stage of fibrosis and grade of activity are shown in the upper panel. The correlation between periportal ductular reaction and stage of fibrosis and grade of activity are shown in the lower panel. $\left({ }^{*} P<0.05\right)$

Table 1 Correlation between centrilobular zone (CTZ)/portal tract (PT) CK7+ ductular reaction and other histologic features of NASH by univariate linear regression analysis in cross-sectional study $(n=90)$

\begin{tabular}{lrc}
\hline Variable & Coefficient & P-value \\
\hline Frequency of CTZ CK7+ ductular reaction & \\
Grade & 0.173 & \\
Stage & 0.154 & $0.0002^{*}$ \\
Steatosis & 0.026 & $0.4989^{*}$ \\
Location of steatosis & -0.009 & 0.886 \\
Lobular inflammation & 0.074 & $0.058^{* *}$ \\
Ballooning & 0.101 & $0.007^{*}$ \\
Mallory body & 0.083 & $0.005^{*}$ \\
Portal inflammation & 0.025 & 0.545 \\
& & \\
Frequency of PT CK7+ductular reaction & \\
Grade & 0.109 & $0.024^{*}$ \\
Stage & 0.111 & $5.27 \mathrm{E}-05^{*}$ \\
Steatosis & -0.005 & 0.906 \\
Location of steatosis & -0.073 & 0.249 \\
Lobular inflammation & -0.009 & 0.830 \\
Ballooning & 0.072 & $0.062^{* *}$ \\
Mallory body & 0.057 & $0.0550^{* *}$ \\
Portal inflammation & 0.112 & $0.005^{*}$ \\
\hline
\end{tabular}

${ }^{*} P<0.05 ;{ }^{*} P<0.1$.

${ }^{a}$ Frequency of CTZ or PT CK7+ ductular reaction: the frequency of any CK7-positive structure in the centrilobular zone or portal tract in a given biopsy. value of 48.7) $(P$-value $=0.08)($ Table 7$)$. However, neither the serum alanine transaminase nor aspartate transaminase level was positively correlated with the frequency of centrilobular ductular reaction in linear regression studies. The aspartate transaminase/ alanine transaminase ratio was slightly higher in the progressor group (0.82) than the non-progressor group (0.64), but the difference was not statistically significant $(P=0.13)$.

\section{Discussion}

In this study we first systematically evaluated the degree of ductular reaction in adult non-alcoholic steatohepatitis biopsies and its relation to stage of fibrosis and grade of activity in a cross-sectional study. A total of 3283 centrilobular zone and portal tracts were analyzed from 90 biopsies that were distributed among the different fibrosis stages (excluding cirrhosis) and necroinflammatory grades. In this study ductular type epithelium was strictly defined as small cells with strong CK7 positivity. Intermediate phenotype hepatocytes with weak CK7 immunoreactivity were carefully excluded to ensure accurate quantitation of unequivocal ductular 
Table 2 Correlation between grade/stage and centrilobular zone (CTZ)/portal tract (PT) CK7+ ductular reaction by multivariate linear regression analysis in cross-sectional study $(n=90)$.

\begin{tabular}{lccc}
\hline Variable & Regression coefficient & S.e. of regression coefficient & T-value \\
\hline Grade & & & \\
Frequency of CTZ CK7+ ductular reaction & 0.770 & 0.250 & 3.078 \\
Frequency of PT CK7+ ductular reaction & 0.152 & 0.248 & $0.003^{*}$ \\
& & & 0.541 \\
Fibrosis stage & & 0.370 & \\
Frequency of CTZ CK7+ ductular reaction & 1.856 & 0.366 & 5.018 \\
Frequency of PT CK7+ ductular reaction & 0.646 & $2.74 \mathrm{e}-06^{*}$ \\
\hline
\end{tabular}

${ }^{*} P<0.05 ;{ }^{*} P<0.1$

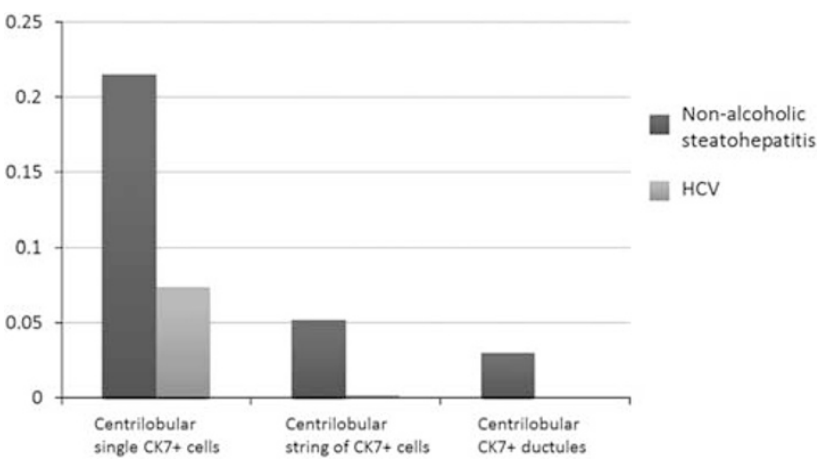

Figure 5 Ductular reaction in HCV hepatitis. The frequency of centrilobular ductular elements in HCV hepatitis patients $(n=19)$ is compared with that in non-alcoholic steatohepatitis patients $\left(n=67 ;{ }^{* *} P<0.05\right)$

Table 3 Change in fibrosis stage between first and last biopsy $(n=47)$

\begin{tabular}{lc}
\hline$n$ & 47 \\
Intervals (years) & $3.2($ range $1.0-8.0)$ \\
Progressors & $21(45 \%)$ \\
Stable & $18(38 \%)$ \\
Regressors & $8(17 \%)$
\end{tabular}

reaction. The centrilobular ductular reaction was scored as single scattered CK7-positive cells, strings of CK7-positive cells, and CK7-positive ductules with lumens, following the stepwise process observed in ductal plate formation during embryogenesis and hepatic reparative complex after massive hepatic injury in experimental models. ${ }^{24}$ The use of glutamine synthetase staining allowed separate analysis of ductular reaction occurring within the periportal and centrilobular compartments.

This study revealed that centrilobular ductular reaction is a common finding in non-alcoholic steatohepatitis and its presence correlated well with lobular inflammation, hepatocellular ballooning degeneration Mallory-Denk bodies, and overall grade of necroinflammatory activity. Fatty acids and their
Table 4 Histologic features at baseline $(n=47)$

\begin{tabular}{|c|c|c|c|c|c|c|c|}
\hline \multicolumn{2}{|c|}{ Fibrosis stage } & \multicolumn{2}{|r|}{ Steatosis } & \multicolumn{2}{|c|}{$\begin{array}{c}\text { Lobular } \\
\text { inflammation }\end{array}$} & \multicolumn{2}{|c|}{$\begin{array}{c}\text { Portal } \\
\text { inflammation }\end{array}$} \\
\hline 0 & $15(32 \%)$ & 1 & 18 (38\%) & 0 & $1(2 \%)$ & 0 & $18(38 \%)$ \\
\hline $1 \mathrm{~A}$ & $10(21 \%)$ & 2 & $16(34 \%)$ & 1 & $24(51 \%)$ & 1 & $15(32 \%)$ \\
\hline 1B & $11(23 \%)$ & 3 & $13(28 \%)$ & 2 & $16(34 \%)$ & 2 & $11(23 \%)$ \\
\hline $1 \mathrm{C}$ & $1(2 \%)$ & & & 3 & $6(13 \%)$ & 3 & $2(4 \%)$ \\
\hline 2 & $6(13 \%)$ & & & & & & \\
\hline 3 & $4(9 \%)$ & & & & & & \\
\hline \multicolumn{2}{|c|}{ Ballooning } & \multicolumn{2}{|c|}{ Mallory bodies } & \multicolumn{2}{|c|}{ Neutrophils } & \multicolumn{2}{|c|}{ Hepatocellular iron } \\
\hline 0 & $2(4 \%)$ & 0 & $32(68 \%)$ & 0 & 27 (57\%) & 0 & $33(70 \%)$ \\
\hline 1 & 30 (64\%) & 1 & $12(26 \%)$ & 1 & $17(36 \%)$ & 1 & $11(23 \%)$ \\
\hline 2 & $15(32 \%)$ & 2 & 3 (6\%) & 2 & $3(6 \%)$ & 2 & 3 (6\%) \\
\hline
\end{tabular}

toxic metabolite accumulate first in the centrilobular zone and thus produce a centrilobular predominant injury pattern in non-alcoholic steatohepatitis. The coincident development of ductular reaction in the centrilobular zones likely represents a direct regenerative/repair response to hepatocyte injury.

Furthermore, this study shows that ductular reaction, and especially centrilobular ductular reaction, was highly correlated with the stage of fibrosis in adult non-alcoholic steatohepatitis in the crosssectional analysis. Centrilobular CK7-positive ductular type epithelium has recently been recognized in patients with chronic hepatic venous outflow obstruction, a condition also featuring a centrilobular predominant injury pattern, presumably due to pressure induced mechanical injury. Pai et al. ${ }^{32}$ reported that the total amount of CK7-positive epithelium (including ductular reaction and intermediate phenotype hepatocytes) correlated well with the degree of fibrosis in biopsies with hepatic venous outflow obstruction. Another study carried out by Krings et al. ${ }^{33}$ further delineated the centrilobular CK7-positive elements into ductular reaction and intermediate phenotype hepatocytes. They found that both correlate well with the stage of fibrosis and interestingly, neither correlate with the amount of periportal ductular reaction. Furthermore, periportal ductular reaction in chronic hepatic 
Table 5 Baseline histology features among patients with progressive and non-progressive fibrosis

\begin{tabular}{|c|c|c|c|}
\hline & Progressor & Non-progressor & P-value \\
\hline & $n=21$ & $n=26$ & \\
\hline \multicolumn{4}{|l|}{ Histology parameters } \\
\hline Fibrosis stage & 0.952 & 0.808 & 0.603 \\
\hline Degree of steatosis & 1.905 & 1.885 & 0.905 \\
\hline Location of steatosis, centrilobular & $65.0 \%$ & $76.9 \%$ & 0.984 \\
\hline Lobular inflammation & 1.667 & 1.500 & 0.379 \\
\hline Neutrophils & 0.667 & 0.346 & 0.116 \\
\hline Ballooning & 1.381 & 1.231 & 0.401 \\
\hline Mallory body & 0.429 & 0.345 & 0.810 \\
\hline Portal inflammation & 1.000 & 0.846 & 0.610 \\
\hline Iron & 0.429 & 0.346 & 0.453 \\
\hline \multicolumn{4}{|l|}{ CK 7 scoring } \\
\hline Frequency of centrilobular CK7+ ductular reaction (sum) & 0.45 & 0.19 & $0.001^{*}$ \\
\hline Centrilobular single cells (\%) & 0.303 & 0.171 & $0.056^{* *}$ \\
\hline Centrilobular strings (\%) & 0.067 & 0.01 & $0.004 *$ \\
\hline Centrilobular ductules (\%) & 0.08 & 0.0085 & 0.057 ** \\
\hline Frequency of Portal tract CK7+ductular reaction (sum) & 0.68 & 0.619 & 0.519 \\
\hline Portal tract mild DR (\%) & 0.331 & 0.339 & 0.897 \\
\hline Portal tract florid DR (\%) & 0.35 & 0.28 & 0.457 \\
\hline
\end{tabular}

${ }^{*} P<0.05 ;{ }^{*} P<0.1$

Table 6 Predictors of fibrosis rate by univariate nonlinear regression analysis

\begin{tabular}{lcc}
\hline Variable & $\begin{array}{c}\text { Regression } \\
\text { coefficient }\end{array}$ & P-value \\
\hline Steatosis & -0.028 & 0.779 \\
Location of steatosis & -0.057 & 0.748 \\
Lobular inflammation & -0.089 & 0.418 \\
Neutrophils & 0.116 & 0.374 \\
Ballooning & 0.155 & 0.261 \\
Mallory body & 0.100 & 0.452 \\
Portal inflammation & -0.373 & 0.677 \\
Iron & 0.042 & 0.726 \\
Frequency of centrilobular CK7+ & 0.630 & $0.020^{* *}$ \\
ductular reaction & & \\
Frequency of portal tract CK7+ & 0.050 & 0.844 \\
ductular reaction & & \\
\hline
\end{tabular}

${ }^{* *} P<0.05$.

venous obstruction did not correlate with degree of fibrosis in both studies.

Periportal ductular reaction is also a frequent finding in non-alcoholic steatohepatitis, and the degree of periportal ductular reaction has been reported to correlate well with fibrosis stage in earlier studies. ${ }^{18,19}$ Therefore, it has been postulated that multiple fibrogenic pathways are involved in non-alcoholic steatohepatitis. Activation of sinusoidal hepatic stellate cells induced by hepatocellular injury and lobular inflammation lead to transformation into myofibroblasts, which then produce collagen along the sinusoids, resulting in centrilobular fibrosis. The periportal ductular reaction is regarded as an independent event induced by a yet unclear mechanism and results in the development of portal and periportal fibrosis. The results from our study did reveal a positive correlation between periportal ductal reaction and the stage of fibrosis. However, this association is much weaker when analyzed together with centrilobular ductular reaction in a multivariate analysis. Our results could be interpreted to argue that centrilobular ductular reaction may be an important driving force in the postulated ductular reaction-promoted fibrogenic pathway in non-alcoholic steatohepatitis, while periportal ductular reaction may represent a more general and less specific response.

The most important finding of this study is that only the frequency of centrilobular ductular reaction differs significantly between the groups of fibrosis progressors and non-progressors, and is the only histologic findings that correlates with fibrosis progression. Previous studies have also shown that none of the routinely reported histologic parameters in non-alcoholic steatohepatitis correlates with fibrosis progression in the future. ${ }^{5,7-17}$ Assessment of centrilobular ductular reaction in pathology reporting of non-alcoholic steatohepatitis may help identifying the rapid progressors who require more aggressive medical intervention and life style changes. The major limitation of this study is the relatively small sample size and short follow-up time. Confirmation of these findings in larger scale studies with longer follow-up time and further simplifying the scoring methods for CK7-positive ductular elements to facilitate clinical use are important next steps of this study.

Diabetes, ${ }^{34}$ body weight, ${ }^{35}$ hypertension, and serum aspartate transaminase/alanine transaminase ratio $^{16}$ are among the clinical factors that have shown correlation with fibrosis progression in previous studies. However, there has been considerable 
Table 7 Clinical parameters

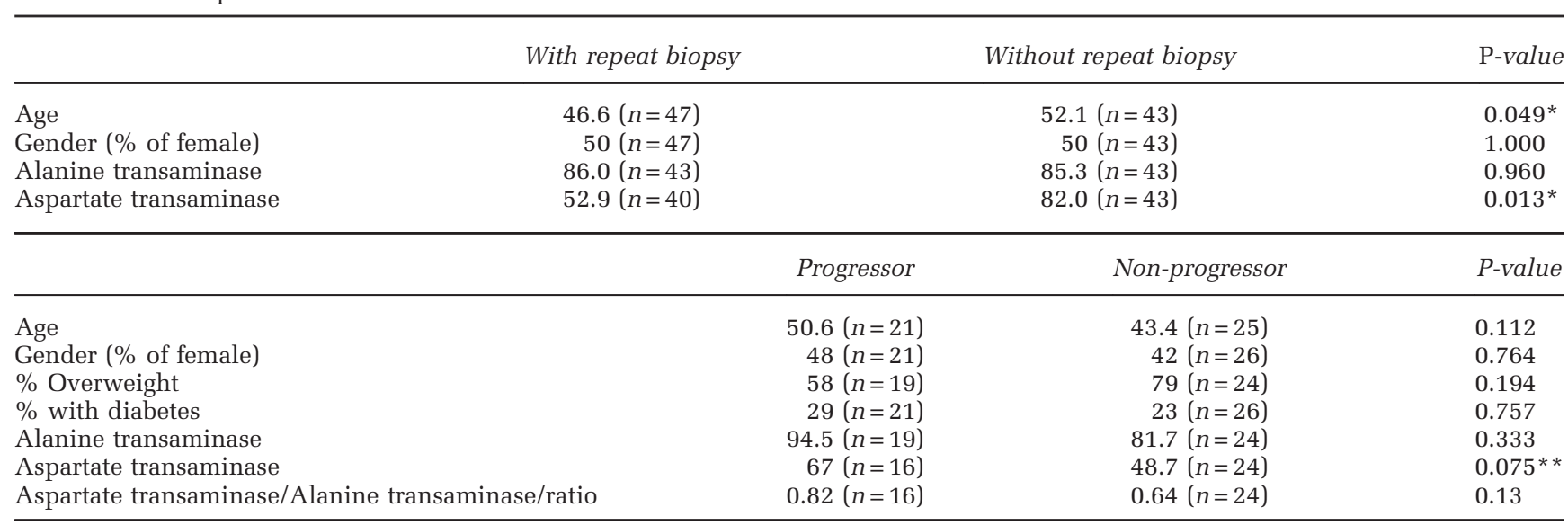

${ }^{*} P<0.05 ;{ }^{*} P<0.1$

heterogeneity in terms of patient populations and diagnostic standards (biopsy proven versus clinical evaluation only) in these studies and the conclusions have been inconsistent. In our analysis, these factors fail to show significant difference between the progressors and non-porgressors. The only parameter that differed significantly was the serum aspartate transaminase level, which on average was higher in progressors than in non-progressors $(P=0.075)$. As this was a retrospective study additional potentially predictive clinical features could not be assessed.

It is beyond the scope of this study to determine the lineage and origin of the ductular reaction in non-alcoholic steatohepatitis. ${ }^{36}$ We speculate that centrilobular ductular reaction in non-alcoholic steatohepatitis is mechanistically similar to that proposed in hepatic venous outflow obstruction (ductular reaction type 2B), ${ }^{37}$ namely, a dedifferentiation of injured hepatocytes back to a progenitor cell-like phenotype. However, significant morphologic differences between the two conditions does exist. The centrilobular ductular reaction in hepatic venous obstruction often occurs within an extensive background of intermediate phenotype CK7-positive hepatocytes, whereas the centrilobular ductular reaction in non-alcoholic steatohepatitis is more focal and is not accompanied by intermediate phenotype hepatocytes. Migration of hepatic progenitor cells into the centrilobular zone is also a possibility. But as discussed above, a general expansion of portal hepatic progenitor cells and portal ductular reaction themselves seem to be a less specific phenomenon. Other possible origins of ductular reaction that have been proposed in the literature includes bone marrow derived progenitor cells or stellate cells that have undergone mesenchymal epithelial transformation. ${ }^{38,39}$

In summary, this study investigated the prevalence of centrilobular and periportal ductular reaction in non-alcoholic steatohepatitis and its relation to disease activity and fibrosis. Centrilobular ductular reaction in non-alcoholic steatohepatitis is a previously under-recognized morphologic finding which may play a key role in non-alcoholic steatohepatitis fibrogenesis. Our study shows that the degree of centrilobular ductular reaction may serve as a histologic marker to predict the development of future fibrosis.

\section{Acknowledgments}

The authors thank Shu-Yuan Xiao for critical reading of the manuscript, Jun Ma for statistical analysis, and Dorothy Kane for technical support.

\section{Disclosure/conflict of interest}

The authors declare no conflict of interest.

\section{References}

1 Neuschwander-Tetri BA, Clark JM, Bass NM, et al. Clinical, laboratory and histological associations in adults with nonalcoholic fatty liver disease. Hepatology 2010;52:913-924.

2 Brunt EM, Janney CG, Di Bisceglie AM, et al. Nonalcoholic steatohepatitis: a proposal for grading and staging the histological lesions. Am J Gastroenterol 1999;94: 2467-2474.

3 Brunt EM. Nonalcoholic steatohepatitis. Semin Liver Dis 2004;24:3-20.

4 Kleiner DE, Brunt EM, Van Natta M, et al. Design and validation of a histological scoring system for nonalcoholic fatty liver disease. Hepatology 2005;41: 1313-1321.

5 Argo CK, Caldwell SH. Epidemiology and natural history of non-alcoholic steatohepatitis. Clin Liver Dis 2009;13:511-531.

6 Angulo P. Long-term mortality in nonalcoholic fatty liver disease: is liver histology of any prognostic significance? Hepatology 2010;51:373-375.

7 Fassio E, Alvarez E, Domínguez N, et al. Natural history of nonalcoholic steatohepatitis: a longitudinal study of repeat liver biopsies. Hepatology 2004;40:820-826. 
8 Adams LA, Sanderson S, Lindor KD, et al. The histological course of nonalcoholic fatty liver disease: a longitudinal study of 103 patients with sequential liver biopsies. J Hepatol 2005;42:132-138.

9 Ekstedt M, Franzén LE, Mathiesen UL, et al. Long-term follow-up of patients with NAFLD and elevated liver enzymes. Hepatology 2006;44:865-873.

10 Hui AY, Wong VW, Chan HL, et al. Histological progression of non-alcoholic fatty liver disease in Chinese patients. Aliment Pharmacol Ther 2005;21:07-413.

11 Harrison S, Torgerson S, Hayashi PH, et al. The natural history of nonalcoholic fatty liver disease: a clinical histopathological study. Am J Gastroenterol 2003;98: 2042-2047.

12 Stepanova M, Rafiq N, Makhlouf H, et al. Predictors of all-cause mortality and liver-related mortality in patients with non-alcoholic fatty liver disease (NAFLD). Dig Dis Sci 2013;58:3017-3023.

13 Younossi ZM, Stepanova M, Rafiq N, et al. Pathologic criteria for nonalcoholic steatohepatitis: interprotocol agreement and ability to predict liver-related mortality. Hepatology 2011;53:1874-1882.

14 Söderberg, Stål P, Askling J, et al. Decreased survival of subjects with elevated liver function tests during a 28year follow-up. Hepatology 2010;51:595-602.

15 McPherson S, Hardy T, Henderson E, et al. Evidence of NAFLD progression from steatosis to fibrosingsteatohepatitis using paired biopsies: implications for prognosis and clinical management. J Hepatol 2014;62: 1148-1155.

16 Singh S, Allen AM, Wang Z, et al. Fibrosis progression in nonalcoholic fatty liver vs nonalcoholic steatohepatitis: a systematic review and meta-analysis of pairedbiopsy studies. Clin Gastroenterol Hepatol 2015;13: 643-654.

17 Wong VWS, Wong GLH, Choi PCL, et al. Disease progression of non-alcoholic fatty liver disease: a prospective study with paired liver biopsies at 3 years. Gut 2010;59:969-974.

18 Richardson MM, Jonsson JR, Powell EE, et al. Progressive fibrosis in nonalcoholic steatohepatitis: association with altered regeneration and a ductular reaction. Gastroenterology 2007;133:80-90.

19 Skoien R, Richardson MM, Jonsson JR, et al. Heterogeneity of fibrosis patterns in non-alcoholic fatty liver disease supports the presence of multiple fibrogenic pathways. Liver Int 2013;33:624-632.

20 Gill RM, Belt P, Wilson L, et al. Centrizonal arteries and microvessels in nonalcoholic steatohepatitis. Am J Surg Pathol 2011;35:1400-1404.

21 Roskams TA, Theise ND, Balabaud C, et al. Nomenclature of the finer branches of the biliary tree: canals, ductules, and ductular reactions in human livers. Hepatology 2004;39:1739-1745.

22 Gouw ASH, Clouston AD, Theise ND. Ductular reactions in human liver: diversity at the interface. Hepatology 2011;54:1853-1863.

23 Sedlaczek N, Jia JD, Bauer M, et al. Proliferating bile duct epithelial cells are a major source of connective tissue growth factor in rat biliary fibrosis. Am J Pathol 2001;158:1239-1244.

24 Strazzabosco M, Fabris L. Development of the bile ducts: essentials for the clinical hepatologist. J Hepatol 2012;56:1159-1170.

25 Lowes KN, Brennan BA, Yeoh GC, et al. Oval cell numbers in human chronic liver diseases are directly related to disease severity. Am J Pathol 1999;154: 537-541.

26 Clouston AD, Powell EE, Walsh MJ, et al. Fibrosis correlates with a ductular reaction in hepatitis $\mathrm{C}$ : roles of impaired replication, progenitor cells and steatosis. Hepatology 2005;41:809-818.

27 Clouston AD, Jonsson JR, Powell EE. Hepatic progenitor cell-mediated regeneration and fibrosis: chicken or egg? Hepatology 2009;49:1424-1426.

28 Matsukuma S, Takeo H, Kono T, et al. Aberrant cytokeratin 7 expression of centrilobular hepatocytes: a clinicopathological study. Histopathology 2012;61:857-862.

29 Gebhardt R, Tanaka T, Williams GM. Glutamine synthetase heterogeneous expression as a marker for the cellular lineage of preneoplastic and neoplastic liver populations. Carcinogenesis 1989;10:1917-1923.

30 Lamers WH, Vermeulen JL, Hakvoort TB, et al. Expression pattern of glutamine synthetase marks transition from collecting into conducting hepatic veins. J Histochem Cytochem 1999;47:1507-1512.

31 Fleming KE, Wanless IR. Glutamine synthetase expression in activated hepatocyte progenitor cells and loss of hepatocellular expression in congestion and cirrhosis. Liver Int 2013;33:525-534.

32 Pai RK, Hart JA. Aberrant expression of cytokeratin 7 in perivenular hepatocytes correlates with a cholestatic chemistry profile in patients with heart failure. Modern Pathol 2010;23:1650-1656.

33 Krings G, Can B, Ferrell L. Aberrant centrizonal features in chronic hepatic venous outflow obstruction: centrilobular mimicry of portal-based disease. Am J Surg Pathol 2014;38:205-214.

34 Loomba R, Abraham M, Unalp A, et al. Association between diabetes, family history of diabetes, and risk of nonalcoholic steatohepatitis and fibrosis. Hepatology 2012;56:943-951.

35 Marchesini G, Petta S, Dalle Grave R. Diet, weight loss, and liver health in nonalcoholic fatty liver disease: Pathophysiology, evidence, and practice. Hepatology 2016;63:2032-2043.

36 Turányi E, Dezs“o K, Csomor J, et al. Immunohistochemical classification of ductular reactions in human liver. Histopathology 2010;57:607-614.

37 Desmet VJ. Ductal plates in hepatic ductular reactions. Hypothesis and implications. I. Types of ductular reaction reconsidered. Virchows Arch 2011;458:251-259.

38 Itoh T, Miyajima A. Liver regeneration by stem/ progenitor cells. Hepatology 2014;59:1617-1626.

39 Sicklick JK, Choi SS, Bustamante M, et al. Evidence for epithelial-mesenchymal transitions in adult liver cells. Am J Physiol Gastrointest Liver Physiol 2006;291: G575-G583.

Supplementary Information accompanies the paper on Modern Pathology website (http://www.nature.com/ modpathol) 